\title{
Space-charge-limited current in cylindrical diodes with finite-length emitter
}

\author{
K. G. Kostov a) and J. J. Barroso \\ Associated Plasma Laboratory, National Institute for Space Research-INPE, 12201-970 São José dos \\ Campos, SP, Brazil
}

(Received 28 March 2001; accepted 3 December 2001)

\begin{abstract}
In the present paper we extend to two dimensions the classical Langmuir-Blodgett law, which determines the maximum current $I_{\mathrm{LB}}$ that can be accelerated by a given voltage across two infinitely long coaxial cylinders. Generalization of the $I_{\mathrm{LB}}$ law is established by performing two-dimensional 2D particle-in-cell numerical experiments on a variety of cylindrical diode configurations with a finite-length emitter. It is found that the limiting current in two dimensions $I_{2 \mathrm{D}}$ follows a monotonically decreasing function of the emitter length-to-outer electrode radius ratio $L / R$ as expressed by the fitting function $I_{2 \mathrm{D}} / I_{\mathrm{LB}}=1+0.1536 /(L / R)+0.0183 /(L / R)^{2}$ within $2.5 \%$ accuracy for simulation data in which the ratio of the outer to inner radii exceeds 3. (C) 2002 American Institute of Physics. [DOI: 10.1063/1.1446876]
\end{abstract}

The effect of space charge on the flow of charged particles between two parallel electrodes in vacuum is of great importance in microwave electronics, crossed-field devices, and high-power diodes. In particular, if too much charge is injected into a gap, the resulting electric field becomes sufficiently high to reflect the injected particles back to the emitter, forming a virtual cathode. Limiting currents of vacuum diodes are classical subjects with early works dating back to the beginning of the last century when onedimensional space-charge-limited flow solutions were derived. Only for simple cases, however, such as plane parallel plates, infinitely long coaxial cylinders, and concentric sphere there exist analytical solutions. ${ }^{1}$ For a planar gap with gap separation $D$ and gap voltage $V$, the maximum electron current density in a space-charge-limited diode is given by the well-known Child-Langmuir law, ${ }^{2}$

$$
j=\frac{4 \epsilon_{0}}{9 D^{2}} \sqrt{\frac{2 e}{m}} V^{3 / 2},
$$

where $e$ and $m$ are, respectively, the charge and the mass of the emitted particles, and $\epsilon_{0}$ is the free space permittivity. Equation (1) is derived by assuming zero electron emission velocity and neglecting relativistic effects. Later an analytical solution for limiting current of relativistic electron flow in the planar diode was found, ${ }^{3}$ but with the generalization of Child-Langmuir law in two dimensions still remaining a formidable task analytically. So an alternative approach was adopted, namely, the use of particle-in-cell (PIC) computer simulation to investigate two-dimensional space-chargelimited flow in a planar diode. ${ }^{4,5}$ About works on cylindrical diodes, particle simulation method was successfully applied for studying the stability of divergent and convergent electron flows in 1D cylindrical Pierce diode. ${ }^{6}$ Also numerical

\footnotetext{
${ }^{a)}$ Also at the Department of General Physics, Sofia University, 5, J. Bourchier Blvd., Sofia-1164, Bulgaria.
}

experiments were performed to examine electrical properties of charged-particle flows in high-power cylindrical pinchedbeam diode over a wide range of voltages. ${ }^{7}$

The electron flow between coaxial cylinders is another important topic on beam formation theory, which has laid the basis of many useful gun designs. High-power cylindrical diodes have also found application in cylindrical vircators ${ }^{8}$ and in high-resolution radiography sources. ${ }^{9}$ Again, as for the planar diode, the specialized literature proposes no analytical solutions for the space-charge-limited current in cylindrical diodes, but in the idealized case of $1 \mathrm{D}$ rectilinear flow between long coaxial cylinders. The solution was given by Langmuir and Blodgett ${ }^{10}$ in terms of an infinite series to calculate the relevant flow parameters. The space-chargelimited electron current per unit length $J_{1 \mathrm{D}}$ at some radial position $r$ between two infinitely long coaxial cylinders is

$$
J_{1 \mathrm{D}} \equiv \frac{I_{\mathrm{LB}}}{L}=\frac{8 \pi \epsilon_{0}}{9} \sqrt{\frac{2 e}{m}} \frac{V^{3 / 2}}{r \beta^{2}},
$$

where $V$ is the potential at a given point, and $\beta=f\left(r / r_{c}\right)$ is a function expressed by the infinite series,

$$
\beta=\mu-\frac{2 \mu^{2}}{5}+\frac{11 \mu^{3}}{120}-\frac{47 \mu^{4}}{3300}+\cdots, \quad \text { with } \mu=\ln \frac{r}{r_{c}} .
$$

Here $r_{c}$ denotes the radius of the emitting electrode. The values of $\beta^{2}$ as a function of $r / r_{c}$ for a cylindrical diode were calculated with precision on the order of $1 \%$ and displayed in table ${ }^{10}$ and graphical ${ }^{7}$ formats.

In the present paper we deal with 2D PIC computer simulation of space-charge-limited current in cylindrical diodes by investigating how Lagmuir-Blodgett law is modified when electrons are emitted only from a finite band over the cathode. The simulation results reported here contribute to enhance our understanding of the operation of a cylindrical diode with a finite emitting band working at a lowvoltage, low-current regime.

Unlike in the planar diode, the electron current between coaxial cylinders depends on the polarity of the electrodes, 


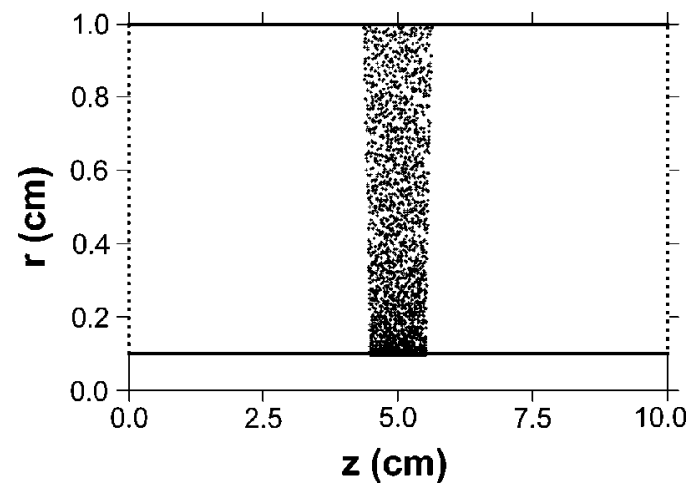

FIG. 1. Schematic of cylindrical diode for PIC simulation with emitter length $L=1.0 \mathrm{~cm}$.

i.e., on the direction of the electron flow. Electrons are emitted from the negative cathode and collected at the anode, which is usually grounded. If the internal electrode of a cylindrical diode serves as cathode while the external cylinder is grounded, the electron flow goes radially outward (divergent flow). In the opposite case-anode inside the cathodethe electrons move inward and the electron flow is convergent. At the same accelerating voltage, both kinds of electron flow in cylindrical diodes carry currents of different intensities, which in the one-dimensional limit (when the length of coaxial electrodes significantly exceeds their radius) can be calculated using formula (2) together with tabulated values of $\beta^{2}$.

To simulate two-dimensional space-charge-limited electron flow in cylindrical diode we used the PIC computer code KARAT. ${ }^{11}$ Numerical simulations proceeded in the electrostatic mode (the beam self-magnetic field is ignored) by using a mesh size of $0.1 \mathrm{~mm}$ in radial direction and $0.2 \mathrm{~mm}$ in the axial direction with a time step of 0.33 ps. The simulation region consists of two $10-\mathrm{cm}$ long, open-ended coaxial cylinders with radii $r$ and $R$. Here $r$ denotes the radius of the internal cylinder and $R$ defines the radius of the external electrode. Both parameters $r$ and $R$ as well as the electrode polarity were varied in the simulation. The cathode is biased at a negative voltage of $1 \mathrm{kV}$ while the anode is grounded. The electrons are emitted from a finite band of length $L$ ( $L$ $\leqslant 5 \mathrm{~cm}$ ) centered at the cathode midsection, as illustrated in Fig. 1.

Currently there exist three different techniques for modeling space-charge-limited (SCL) emission that give quite similar results for the total SCL current in a planar diode. ${ }^{5}$ For the purpose of our simulation we use the so-called overinjection method. The emission current in this model is set uniform over the emitter and made to increase linearly in time from zero to its nominal value for $1 \mathrm{~ns}$ after that it is kept constant during the simulation. The electrons are emitted at the initial energy of $0.1 \mathrm{eV}$. If the injected current exceeds the true limiting current of the diode a virtual cathode will form. The method of overinjection requires a series of simulation in order to find the greatest value of the injection current that does not lead to the formation of a virtual cathode. Although the overinjection method has some disadvantages - in that on the assumption of spatially uni-

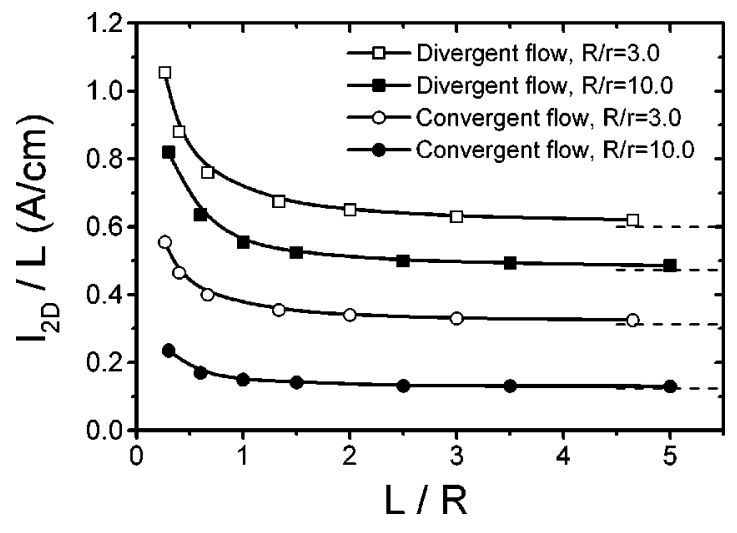

FIG. 2. Space-charge-limited currents per unit length of the convergent and divergent electron flows as functions of the normalized emitter length $L / R$ for simulations with $R / r=3.0(R=1.5 \mathrm{~cm}$ and $r=1.0 \mathrm{~cm})$ and $R / r=10.0$ $(R=1.0 \mathrm{~cm}$ and $r=0.1 \mathrm{~cm})$. The dashed lines on the right side of the curves represent the corresponding $1 \mathrm{D}$ classical limits.

form electron emission it misses the spikes in the current density at the beam edges, as is shown for a planar diode ${ }^{5}$ - this method provides useful estimates and can be easily applied for cylindrical geometry.

For each particular diode configuration (selected $r, R$, and electrode polarity) the simulation starts by specifying the emitting band $L$ and a low injected current $I$. Then the value of current is gradually increased until a virtual cathode is formed. It is located near the cathode surface in the center of the emitting band where the potential depression created by the space charge has a maximal value. The electric field at this point reverses direction reflecting the emitted electrons back to the emitter. The simulation proceeds by successively lowering the injected current until no electron reflection occurs (this is detected by the lack of reflected particles at the cathode). This value of $I / L$ - the maximal injected current per unit length without formation of a virtual cathode-is taken to be the $2 \mathrm{D}$ limiting current per unit length, $J_{2 \mathrm{D}}$, of the cylindrical diode. The results for two diode configurations ( $r=0.1 \mathrm{~cm}, R=1.0 \mathrm{~cm}$ and $r=0.5 \mathrm{~cm}$ and $R=1.5 \mathrm{~cm}$ ) are shown in Fig. 2 where the injected current per unit length is plotted as a function of the dimensionless variable $L / R$. The diode critical currents for convergent and divergent electron flows monotonically increase with decreasing emission length $L$. The straight dashed lines on the right side of Fig. 2 represent the classical 1D space-charge-limited currents per unit length. One can see that for both the diodes presented the 2D limiting currents from the simulation closely approach the $1 \mathrm{D}$ theoretical limit given by (2) as $L / R \geqslant 5$. This result is confirmed for a variety of diode configurations and nonrelativistic gap voltages.

To summarize the numerical data obtained for different diode configurations, the space-charge-limited currents obtained by the simulation are normalized to the corresponding 1D classical limiting currents and the result versus $L / R$ is plotted in Fig. 3. This clearly demonstrates that for all parameters used the normalized current exhibits a general behavior, in principle, similar to that of the $2 \mathrm{D}$ planar diode. ${ }^{4,5}$ The normalized space-charge-limited current density is a monotonically decreasing function of $L / R$, recovering the 


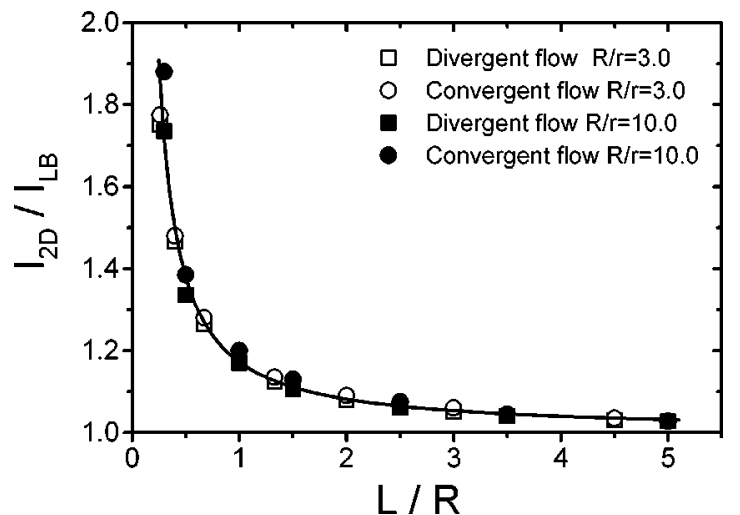

FIG. 3. Two-dimensional limiting current density $J_{2 \mathrm{D}}$ in units of the onedimensional Langmuir-Blodgett value. The symbols correspond to the simulation results and the curve represents the mean-square-root fitting.

classical 1D limiting current as $L / R \geqslant 5$. For both aspect ratios, i.e., $R / r=3$ and $R / r=10$, and over the simulated range $0.2<L / R<5$ the normalized numerical data for $2 \mathrm{D}$ spacecharge-limited current may be fitted empirically by a function of the single variable $L / R$,

$$
\frac{I_{2 \mathrm{D}}}{I_{\mathrm{LB}}}=1+\frac{0.1536}{L / R}+\frac{0.0183}{(L / R)^{2}},
$$

to within $2.5 \%$ for all simulation data.

Since $J_{1 \mathrm{D}}$, via the parameter $\beta^{2}$, is a function of $R / r$ [see Eq. (2)], one could expect that the 2D space-chargelimited current in cylindrical geometry will be a function of two parameters-the normalized emitter length $L / R$ and diode aspect ratio $R / r$. However, as Fig. 3 shows over the simulation range $R / r$ from 3 to 10 , the numerical data can be well empirically fitted by a function of only one variable $L / R$. This result can be understood if we look at the values of $\beta^{2}$, which for $R / r>3$ rapidly approach its asymptotic value. ${ }^{1,7}$ However, for smaller ratios $(R / r<3)$ the behavior of $\beta^{2}$ drastically changes as it quickly decreases to zero and the fitting (3) is no longer valid.

Further physical insight into 2-D features of the spacecharge-limited flow in the cylindrical diode is provided by the axial distribution of the current per unit length $I / L$. In the one-dimensional limit, the electron motion is considered to be rectilinear so the current density is uniform along the diode axis. However, in the case of a finite emitting band the beam expands in the transversal (to the field lines) direction due to the effect of space charge such that the current density distribution along the axis is no longer uniform. The total beam current that reaches the anode in this case is obtained after integrating the current distribution function. Figure 4 shows typical distribution functions of the space-chargelimited current per unit length $I / L$ on the anode of cylindrical diodes with $R / r=10$ for both kinds of electron flow (divergent and convergent). Roughly, the distribution function has a trapezoidal shape with a maximal value that rises with increasing injected current until the 2D space-charge-limited current is reached.

While having a trapezoidal-like shape with rounded corners, the current profiles in Figs. 4 show a maximal value
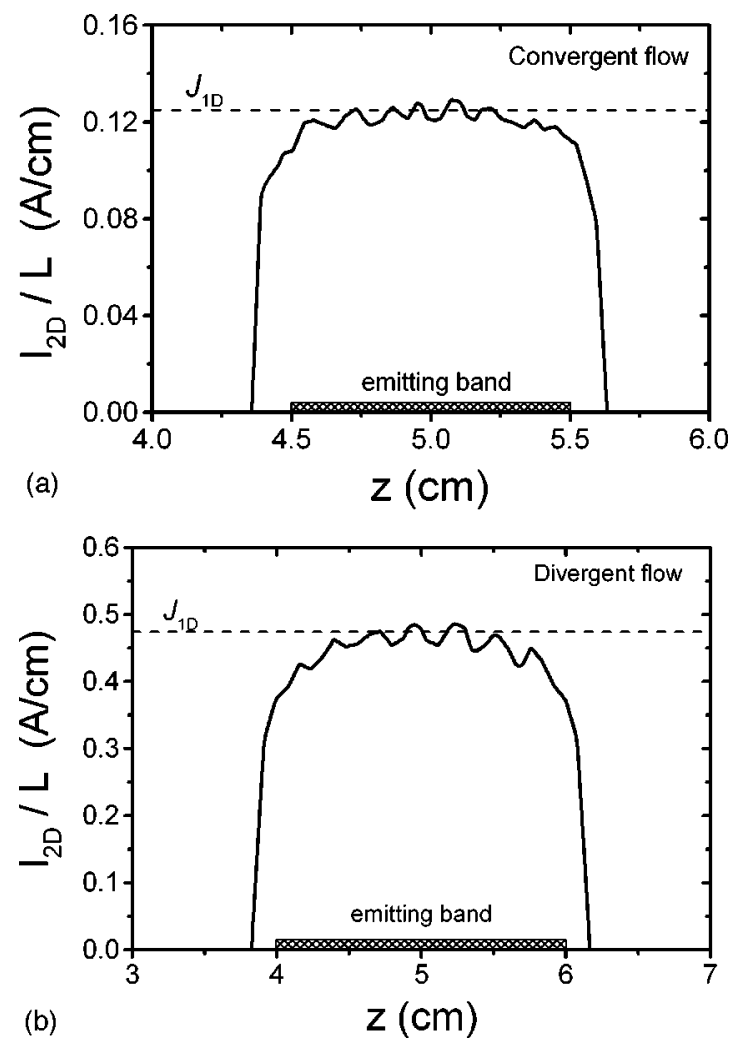

FIG. 4. The axial distribution of the space-charged-limited current density at the anode of cylindrical diode with $R / r=10.0(R=1.0 \mathrm{~cm}, r=0.1 \mathrm{~cm})$. (a) $L=1.0 \mathrm{~cm}$, and 0.16 A convergent electron flow, and (b) $L=2.0 \mathrm{~cm}, 1.0$ A divergent electron flow. The dashed line indicates the limiting current densities $J_{1 \mathrm{D}}$ predicted by Eq. (2). The location of the emitting bands is illustrated by the hatched area.

that closely matches the $1 \mathrm{D}$ classical current per unit length limit $J_{1 \mathrm{D}}$. We emphasize that our simulations have dealt with beams injected at zero initial velocity and accelerated through a magnetic-field-free gap; and on account of the beam spreading, the current density profiles are trapezoidallike with maximal value $J_{1 \mathrm{D}}$ as allowed by one-dimensional theory. Were the beam immersed in a strong magnetic field, then the corresponding current density profile would approach a rectangular profile with maximal value exceeding the classical 1D value; the total collected current, however, would be virtually the same as that in the unconfined flow. This is equivalent to saying that the total current is virtually independent of the external magnetic field $\mathrm{B}$, but the current density profile depends on and correlates with $\mathrm{B}$. In addition, by further increasing the injected current from the maximal 2D value, a virtual cathode develops and the axial current density distribution on the anode abruptly changes its shape. Indicative of virtual cathode formation at the center of the cathode, an indentation appears in the middle of such a current distribution, with amplitude less than the 1D classical limit $J_{1 \mathrm{D}}$.

In summary, the one-dimensional Langmuir-Blodgett formula for the current limited by space charge between coaxial cylinders is generalized to two dimensions via particle simulation based on the overinjection method. The limiting current of the cylindrical diode is found to be a monotonically decreasing function of $L / R$. This function is examined 
over a broad range of diode parameters and for convergent and divergent electron flows. The detailed investigation of the beam current per unit length collected at the anode demonstrates that maximal values of the distribution functions are those predicted by the $1 \mathrm{D}$ classical limit.

\section{ACKNOWLEDGMENT}

This work was supported by the Research Assisting Foundation of the State of São Paulo (FAPESP), Brazil.

${ }^{1}$ P. T. Kirstein, G. S. Kino, and W. E. Waters, Space-Charge Flow (McGraw-Hill, New York, 1967).

${ }^{2}$ C. D. Child, Phys. Rev. 32, 492 (1911); I. Langmuir, ibid. 2, 450 (1913).

${ }^{3}$ H. R. Jory and A. W. Travelpiece, J. Appl. Phys. 40, 3924 (1969).
${ }^{4}$ J. W. Luginsland, Y. Y. Lau, and R. M. Gilgenbach, Phys. Rev. Lett. 77, 4668 (1996).

${ }^{5}$ J. J. Watrous, J. W. Lugisland, and G. E. Sasser III, Phys. Plasmas 8, 289 (2001).

${ }^{6}$ M. V. Alves, F. T. Gratton, G. Gnavi, and C. H. Moreno, Phys. Plasmas 4, 3049 (1997).

${ }^{7}$ S. B. Swanekamp, R. J. Commisso, G. Cooperstein, P. F. Ottinger, and J. W. Schumer, Phys. Plasmas 7, 5214 (2000).

${ }^{8}$ W. Jiang, K. Woolverton, J. Dickens, and M. Kristiansen, IEEE Trans. Plasma Sci. 27, 1538 (1999).

${ }^{9}$ G. Cooperstein, R. J. Commisso, D. D. Hinshelwood, P. F. Ottinger, D. V. Ross, S. J. Stephanakis, S. B. Swanekamp, and F. C. Young, Proceedings of the 12th International Conference on High-Power Beams, Haifa, Israel, edited by M. Markovitz and J. Shiloh (Institute of Electrical, and Electronics Engineering, Piscataway, NJ, 1988), p. 31.

${ }^{10}$ I. Langmuir and K. Blodgett, Phys. Rev. 22, 347 (1923).

${ }^{11}$ V. P. Tarakanov, User's Manual for Code KARAT (Berkeley Research Associates, Inc., Springfield, VA, 1994). 